\title{
Flexural Waves in Fluid-Filled Tubes Subject to Axial Impact
}

\author{
Kazuaki Inaba \\ e-mail: inaba@mech.titech.ac.jp \\ Joseph E. Shepherd \\ e-mail: joseph.e.shepherd@caltech.edu \\ Graduate Aeronautical Laboratories, \\ California Institute of Technology, \\ Pasadena, CA 91125
}

We experimentally studied the propagation of coupled fluid stress waves and tube flexural waves generated through projectile impact along the axis of a water-filled tube. We tested mild steel tubes, 38-40 mm inner diameter and wall thicknesses of $0.8 \mathrm{~mm}, 6.4 \mathrm{~mm}$, and $12.7 \mathrm{~mm}$. A steel impactor was accelerated using an air cannon and struck a polycarbonate buffer placed on top of the water surface within the tube. Elastic flexural waves were observed for impact speeds of 5-10 m/s and plastic waves appeared for impact speeds approaching $20 \mathrm{~m} / \mathrm{s}$ for a $0.8 \mathrm{~mm}$ thickness tube. We observed primary wave speeds of $1100 \mathrm{~m} / \mathrm{s}$ in a $0.8 \mathrm{~mm}$ thickness tube, increasing to the water sound speed with $6.4 \mathrm{~mm}$ and $12.7 \mathrm{~mm}$ thickness tubes. Comparison of our measurements in the $0.8 \mathrm{~mm}$ thickness tube with Skalak's water hammer theory indicates reasonable agreement between the predicted and measured peak strains as a function of the impact buffer speed (1956, “An Extension to the Theory of Water Hammer," Trans. ASME, 78, pp. 105-116). For thick-walled tubes, the correlation between the experimentally determined peak pressures and strains reveals the importance of corrections for the through-wall stress distribution. [DOI: 10.1115/1.4000510]

\section{Introduction}

Impulsive loading and the resulting fluid-structure interaction (FSI) has been extensively studied since WWII $[1,2]$. The classical configuration in these experiments is a flat plate with loading created by the underwater detonation of high explosives at some distance from the plate surface, which results in the normal impact of a shock wave followed by a rapid pressure decay [3]. However, in water hammer events, the FSI is due to the coupling of flexural waves in shells with the pressure (shock) waves in the fluid propagating perpendicular to the surface of the shell. To investigate this type of coupling, we are using projectile impact and water-filled tubes (as shown in Fig. 1).

This configuration is similar to that used by Trevena [4] and more recently by Skews et al. [5], and independently proposed as an underwater shock simulator by Despande et al. [6] and Espinosa et al. [7]. With a piston velocity of $250 \mathrm{~m} / \mathrm{s}$, it is possible to create peak shock pressures of $480 \mathrm{MPa}$ if the tube is considered to be rigid. The actual shock pressure may be significantly lower, depending on the extent of fluid-solid coupling for this configuration. The problem of stress waves propagating in a water-filled tube have been considered extensively in the context of water hammer beginning with Korteweg [8] and Joukowsky [9], and recently reviewed by Wiggert and Tijsseling [10]. There are four axisymmetric modes of deformation for low-amplitude waves [11-13] and the most significant of these for the present study is the Korteweg mode, which is a radial oscillation of the tube coupled to longitudinal motion of the liquid. The extent of fluidsolid coupling in this geometry is determined by the parameter $\mathrm{Ka} /(\mathrm{Eh})$, which unlike the case of normal impact of a shock wave is independent of the flow following the shock and only depends on the fluid and solid properties and geometry.

The simplest theory of the wave propagation in fluid-filled thinwalled tubes is due to Korteweg [8] and was experimentally confirmed by Joukowsky [9]. A much more elaborate and complete theory of this situation was first given by Skalak [14], who treated the tube with shell theory and used an acoustic model to treat the fluid motion. Recent efforts on the theory of water hammer $[10,15-18]$ have concentrated on extending or simplifying

Contributed by the Pressure Vessel and Piping Division of ASME for publication in the Journal of Pressure Vessel Technology. Manuscript received September 29, 2008; final manuscript received June 22, 2009; published online January 29, 2010. Assoc. Editor: Edward A. Rodriguez.
Skalak's theory with some comparison to testing done in piping systems. The main results of Skalak's theoretical treatment are that an initial disturbance such as an impact will generate two waves. The primary or slow wave carries the main disturbance in the fluid pressure and tube strain. The pressure generated by acoustic waves in the fluid is balanced primarily by the hoop stress from the radial motion of the tube. The tube deformation is a radial (hoop) mode coupled to an axial bending mode. The primary wave has a dispersive character, which has been extensively examined by Tijsseling et al. [19].

The precursor or fast wave is a much smaller amplitude (200 times smaller in hoop and 10 times smaller in longitudinal strain) disturbance than the primary wave. The precursor is an almost purely longitudinal strain wave induced by Poisson's effect and the strains associated with the primary wave. Because of the Poisson effect, the hoop motion of the primary wave creates a longitudinal strain in the tube of opposite sign to the hoop strain and a factor of 3-4 smaller in absolute amplitude for the primary wave. The tube deformation associated with the precusor is an axial (bar) wave. The precursor wave was predicted by Skalak [14], taking account of the longitudinal and bending stresses in the pipe wall. If the primary wave has positive pressure and hoop strain, then the precursor will be a longitudinal strain tension wave.

The theories of Korteweg [8] and Skalak [14] are linear and predict that the coupled (elastic) stress waves produced by FSI travel with velocities that are independent of the projectile speed. However, the peak amplitude of the stress waves is predicted to be a linear function of the projectile speed. We have examined these predictions by carrying out experiments over a range of projectile speeds for both thin and thick-walled tubes. Our studies give new data for the regime of plastic deformation and thick-walled tubes.

\section{Experimental Apparatus and Test Procedure}

2.1 Gas Gun. Our tests were carried out using a simple gas gun and specimen tubes filled with water. The facility (Fig. 2) is a low-speed gas gun that is mounted vertically above a specimen tube filled with water. The $0.67 \mathrm{~kg}$ steel projectile is accelerated by a combination of gravity and compressed air using driver (reservoir) pressures, $P_{D}$, between $0.14 \mathrm{MPa}$ and $0.66 \mathrm{MPa}$ (Table 1). Prior to installing the specimen tube, the projectile is loaded into the barrel and using a roughing pump, the projectile is sucked up to the top and held against a rubber seal by the pressure of the air in the barrel. After the specimen tube is aligned and the instru- 


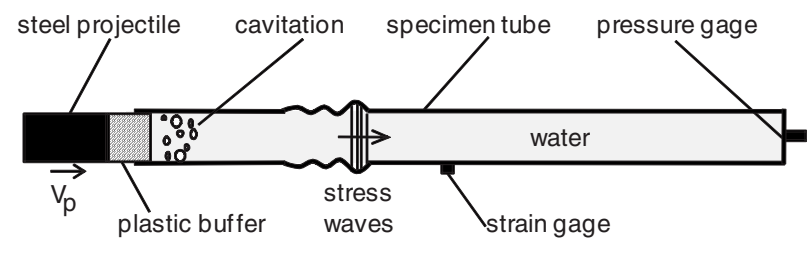

Fig. 1 Schematic diagram of axisymmetric water-in-tube configuration for generation of flexural waves in a shell coupling with stress waves propagating in water

mentation is connected, the projectile is launched down the tube. The air reservoir is filled to the desired pressure, the vacuum line is closed, and a remotely operated valve connects the air reservoir to the evacuated region above the projectile.

The projectile is not completely ejected from the barrel when it impacts a polycarbonate buffer placed on the water surface, which is just inside the specimen tube. A gland seal is used to prevent water moving through the clearance space between the buffer and tube. When the buffer is placed in the tube, the resulting air bubble between the buffer and water free surface is removed

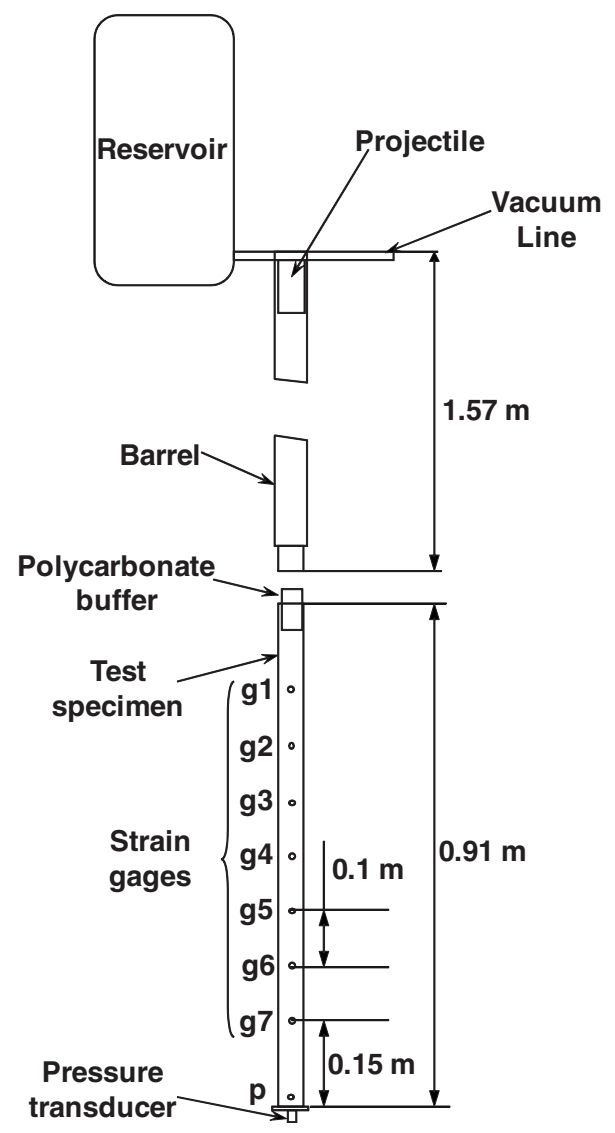

Fig. 2 Experimental facility with reservoir (compressed gas driver), projectile, specimen tube, and gauges

Table 1 Test matrix

\begin{tabular}{ccccc}
\hline \hline Shot & Tube & $\begin{array}{c}P_{D} \\
(\mathrm{MPa})\end{array}$ & $\begin{array}{c}V_{P} \\
(\mathrm{~m} / \mathrm{s})\end{array}$ & $\begin{array}{c}V_{B} \\
(\mathrm{~m} / \mathrm{s})\end{array}$ \\
\hline $17-21$ & 1 & $0.14-0.55$ & $7.8-17.6$ & $6.8-14.0$ \\
28 and 29 & 2 & 0.14 and 0.65 & $9.1,19.3$ & $7.1,16.6$ \\
$30-35$ & 3 & $0.14-0.66$ & $6.7-19.1$ & $5.0-13.4$ \\
$52-56$ & 4 & $0.14-0.66$ & $7.1-19.2$ & $6.8-15.2$ \\
$59-64$ & 5 & $0.14-0.66$ & $6.7-18.5$ & $5.7-15.2$ \\
\hline \hline
\end{tabular}

Table 2 Tube data

\begin{tabular}{lccr}
\hline \hline Tube & $\begin{array}{c}\text { Length } \\
(\mathrm{mm})\end{array}$ & $\begin{array}{c}\text { ID } \\
(\mathrm{mm})\end{array}$ & $\begin{array}{c}h \\
(\mathrm{~mm})\end{array}$ \\
\hline 1 & 915 & 39.7 & 0.77 \\
2 & 906 & 39.9 & 0.77 \\
3 & 905 & 40.0 & 0.77 \\
4 & 908 & 38.0 & 6.44 \\
5 & 908 & 38.1 & 12.74 \\
\hline \hline
\end{tabular}

through a small hole, which is then sealed. In this fashion, the stress waves are transmitted directly to the water surface inside the specimen tube due to the impact of the projectile. This prevents the projectile from impacting the specimen tube directly and enables us to measure the wave velocities without interference from axial waves created by the projectile impact on the tube itself.

The impact generated stress waves in the water cause the tube to deform and the resulting coupled fluid-solid motion propagates down the tube. The deformation of the tube is measured by strain gauges oriented in the hoop and longitudinal directions and the pressure in the water is measured by piezoelectric transducers. In the thin-walled tube, a single pressure transducer is mounted in an aluminum fitting glued to the bottom of the tube. In the thickwalled tube 5, the piezoelectric gauges are mounted on the side of the tubes. The bottom of the tube is fastened to an aluminum bar mounted in a lathe chuck that is placed directly on the floor.

2.2 Test Conditions and Specimens. The three tube configurations tested are listed in Table 2. Shots 17-35 were carried out using thin-walled mild-steel (C1010) tubes (tubes 1-3). The tubes have a wall-thickness of $0.77 \mathrm{~mm}$ and are about $0.91 \mathrm{~m}$ long. Shots 52-56 (tube 4) and 59-64 (tube 5) were carried out with mild-steel (C1010) tubes with wall thicknesses of $6.44 \mathrm{~mm}(0.25$ in.) and $12.74 \mathrm{~mm}(0.5 \mathrm{in}$.). Each test specimen is instrumented with strain gauges at $100 \mathrm{~mm}$ increments; these are gauges $\mathrm{g} 1-\mathrm{g} 7$ in Fig. 2. A high-speed video camera (Vision Research Phantom v.7.3) is used to observe the impact against the buffer and distance-time measurements taken directly from the images were used to determine the speeds of the projectile immediately prior to impact and the surface position of buffer during experiments.

The projectile speed at the exit of the barrel was varied by using different pressures in the gas reservoir. The initial driver gas conditions and measured projectile speeds are given in Table 1. Projectile speeds are determined from video images over $20 \mathrm{~mm}$ of motion. Although there is substantial variability in the exit speed, there is a clear trend of increased projectile speed as reservoir pressure increases. The projectile exit speed is about $5 \mathrm{~m} / \mathrm{s}$ without driver gas and increases with increasing driver pressure; the speed ranges between $6.7 \mathrm{~m} / \mathrm{s}$ and $19.1 \mathrm{~m} / \mathrm{s}$ at barrel exit. Variations in friction, seating of the projectile against the rubber seal, low accuracy of the projectile speed measurement system, and the timing of the filling and discharge process all contributed to the variability in the gun performance.

A position history of the buffer surface is also extracted from movies with MATLAB image processing (Fig. 3). The buffer speed is calculated from the history immediately after the projectile impact. The maximum buffer speeds are observed to consistently be $2-3 \mathrm{~m} / \mathrm{s}$ lower than the projectile impact speeds. The buffer is observed to almost instantly accelerate following the projectile impact but then immediately slows down due to the interaction with water in the tube. Close to the time of arrival of the reflected wave from the tube bottom, the buffer begins to rise and pushes the projectile back up. 


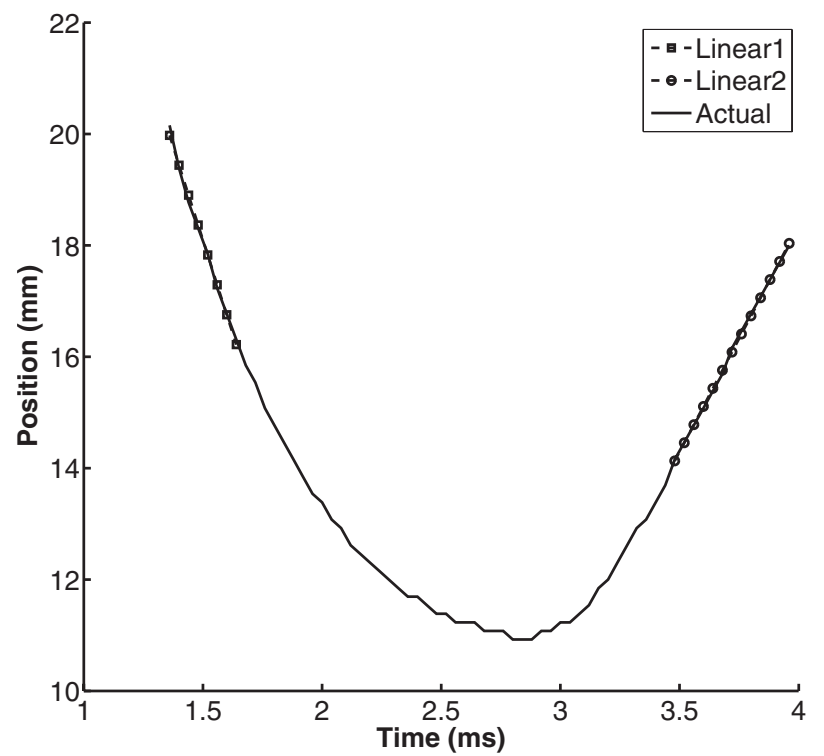

Fig. 3 Buffer surface history in shot $34\left(V_{P}=16.1 \mathrm{~m} / \mathrm{s}, P_{D}\right.$ $=0.64 \mathrm{MPa}$ )

\section{Results and Discussion}

3.1 Elastic Waves in Thin Tubes. Elastic strain waves are excited in the thin-walled tubes when the driver pressure is sufficiently low, e.g., $P_{D}=0.14 \mathrm{MPa}$. Figure 4 shows the hoop-strain histories measured at locations g1 (bottom trace) to g7. The bottom gauge is mounted $21 \mathrm{~mm}$ from the end of the specimen, 129 $\mathrm{m}$ below $\mathrm{g} 7$; the other locations are as given in Fig. 2. The top trace in Fig. 4 is the pressure history measured at the bottom of the specimen tube. In Fig. 4, the line labeled $1213 \mathrm{~m} / \mathrm{s}$ indicates the leading edge of the main stress wave front that is initiated by the impact. The subsequent reflection of stress waves from the bottom and re-reflection from the buffer can be observed as distinct strain pulses in this figure. The averaged peak strain from all seven hoop gauges in shot 28 is $1.19 \mathrm{mstrain}\left(1 \mathrm{mstrain}=10^{-3}\right)$, lower than the nominal proportional elastic limit of $2.0 \mathrm{mstrain}$. The primary wave speed of $1213 \mathrm{~m} / \mathrm{s}$ is about $1 \%$ higher than Skalak's theoretical phase speed, $c_{1}=1199 \mathrm{~m} / \mathrm{s}$. Theoretical values are calculated by using the nominal steel properties and ex-

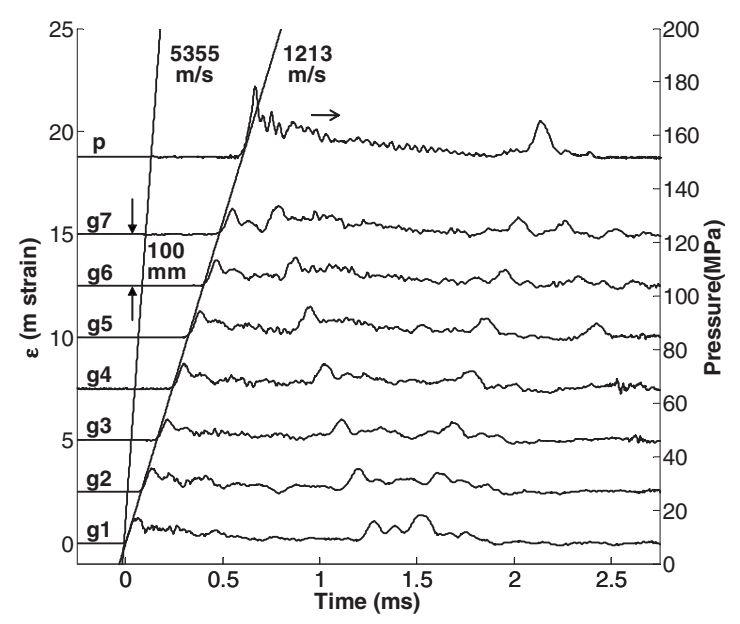

Fig. 4 Hoop strain and end pressure histories-elastic waves in shot $28, V_{B}=7.1 \mathrm{~m} / \mathrm{s}\left(V_{P}=9.1 \mathrm{~m} / \mathrm{s}, P_{D}=0.14 \mathrm{MPa}\right)$
Table 3 Geometrical and material properties (at $295 \mathrm{~K}$ and 1 bar)

\begin{tabular}{lcc}
\hline \hline \multicolumn{2}{c}{ Water } & \\
\hline Bulk modulus & $K$ & $2.200 \mathrm{GPa}$ \\
Density of water & $\rho_{0}$ & $997.7 \mathrm{~kg} / \mathrm{m}^{3}$ \\
Velocity of sound in water & $c$ & $1485 \mathrm{~m} / \mathrm{s}$ \\
& Mild steel (C1010) & \\
Density of steel & $\rho$ & $7860 \mathrm{~kg} / \mathrm{m}^{3}$ \\
Poisson's ratio & $\nu$ & 0.280 \\
Mass of tube per unit surface area & $m$ & $6.01 \mathrm{~kg} / \mathrm{m}^{2}$ \\
Radius of pipe & $a$ & $20.4 \mathrm{~mm}$ \\
Thickness of tube wall & $h$ & $0.77 \mathrm{~mm}$ \\
Young's modulus & $E$ & $211 \mathrm{GPa}$ \\
Tensile strength & & $365 \mathrm{MPa}$ \\
Yield strength & & $305 \mathrm{MPa}$ \\
Elongation & & $20 \%$ \\
\hline \hline
\end{tabular}

perimental geometry listed in Table 3 . The speed $c_{1}$ is $0.4 \%$ higher than the speed $c_{K}=1194 \mathrm{~m} / \mathrm{s}$ predicted by the simple Korteweg theory

$$
c_{K}=\frac{c}{\sqrt{1+\frac{2 K a}{E h}}}
$$

which is often used in the analysis of water hammer events. In agreement with Skalak's theory, a very low amplitude longitudinal precursor in the tube is also observed on the longitudinal strain gauges. The speed is $5355 \mathrm{~m} / \mathrm{s}, 2 \%$ higher than Skalak's theoretical phase speed, $c_{2}=5260 \mathrm{~m} / \mathrm{s}$. The speed $c_{2}$ is $2.9 \%$ lower than the thin-plate longitudinal wave speed in the tube wall $c_{0}$ $=5416 \mathrm{~m} / \mathrm{s}$, given by

$$
c_{0}=\sqrt{\frac{E h}{m\left(1-\nu^{2}\right)}}
$$

The longitudinal strains measured at four locations are shown in Fig. 5. The longitudinal strain wave has the opposite sign as the hoop-strain wave but the absolute amplitude varies in a similar fashion with time. Figure 6 is an enlarged view of an initial portion of the hoop-strain history for shot 28 at g5 in Fig. 2 (the fifth history from the bottom in Fig. 4). In this figure, the longitudinal strain measured at the axial location is also presented. The average values of the peak hoop and longitudinal strains listed in Table 4

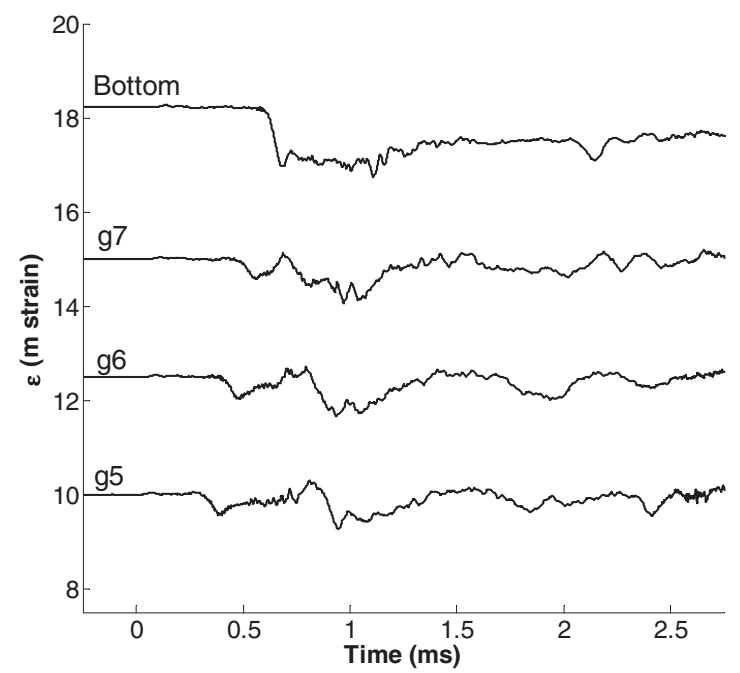

Fig. 5 Longitudinal strain histories-elastic waves in shot 28 


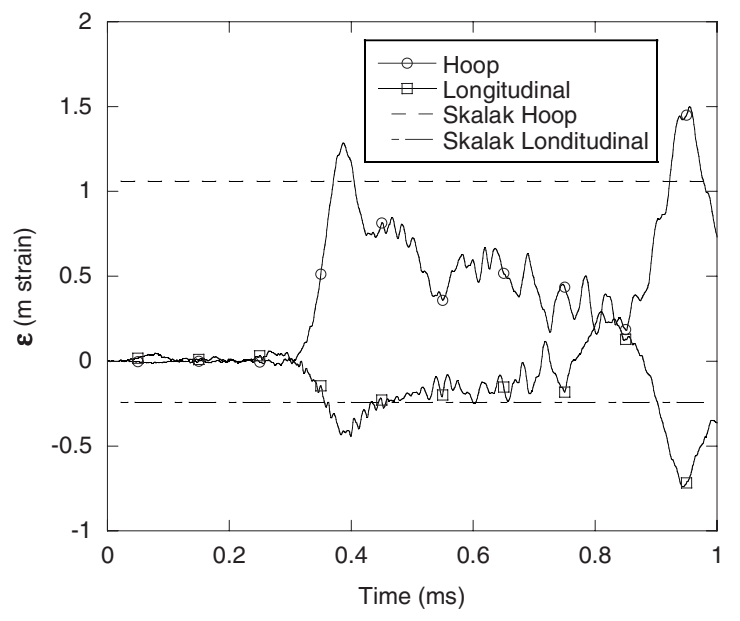

Fig. 6 Hoop and longitudinal strain histories-elastic waves in shot 28, gauge location $5(350 \mathrm{~mm}$ from the bottom of specimen tube $), V_{B}=7.1 \mathrm{~m} / \mathrm{s}\left(V_{P}=9.1 \mathrm{~m} / \mathrm{s}, P_{D}=0.14 \mathrm{MPa}\right)$

are $1.19 m$ strain and $-0.449 m$ strain. Skalak's theory for the hoop strain in the case of sudden stoppage of flow can be applied to the present case and predicts

$$
\varepsilon_{\text {hoop }}=0.792 \frac{p_{0} a}{E h}
$$

where $p_{0}=\rho_{0} V_{B} c$ which gives $\varepsilon_{\text {hoop }}=1.06 m$ strain using the buffer speed, $V_{B}$. The predicted value of peak hoop strain is in reasonable (within 20\%) agreement with the measured values. Using the projectile speed, $V_{P}$, to determine $P_{0}$ results in a prediction of $1.35 \mathrm{~m}$ strain, which is slightly higher than the experimental results. Skalak's theory for the longitudinal strain predicts that

$$
\varepsilon_{\text {longitudinal }}=-0.182 \frac{p_{0} a}{E h}
$$

The predicted values are $-0.243 \mathrm{~m}$ strain and $-0.312 \mathrm{~m}$ strain with $V_{B}$ and $V_{P}$, respectively. The average measured peak value is $-0.449 \mathrm{~m}$ strain, about $50 \%$ larger than the prediction.

Since the buffer speed gradually falls and eventually reverses following the impact, the resulting strain waves have a sharp rise followed by a rapid decay. The decay continues until the reflected wave returns from the bottom of the tube. The decay of pressure and strain behind the initial peak is approximately exponential; this is due to the inertia of the water and the increasing mass of water that must be accelerated by the buffer as the sound waves propagate away from the buffer into the fluid. For an idealized impact problem, we can predict the pressure profile by making use of the classical treatments of shock wave generation and decay in solids [20,21]. For the simplest case, numerical [5] or analytical

Table 4 Experimental data of frontal peak strains in shots 28 and 29

\begin{tabular}{lcc}
\hline \hline Gauge & $\begin{array}{c}\text { Shot 28 } \\
(m \text { strain })\end{array}$ & $\begin{array}{c}\text { Shot 29 } \\
(m \text { strain })\end{array}$ \\
\hline Hoop g1 & 1.24 & 3.06 \\
Hoop g2 & 1.13 & 2.05 \\
Hoop g3 & 1.01 & 1.59 \\
Hoop g4 & 1.20 & 1.85 \\
Hoop g5 & 1.29 & 1.94 \\
Hoop g6 & 1.24 & 1.83 \\
Hoop g7 & 1.24 & 1.79 \\
Longitudinal g5 & -0.443 & -0.749 \\
Longitudinal g6 & -0.472 & -0.799 \\
Longitudinal g7 & -0.432 & -0.684 \\
\hline \hline
\end{tabular}

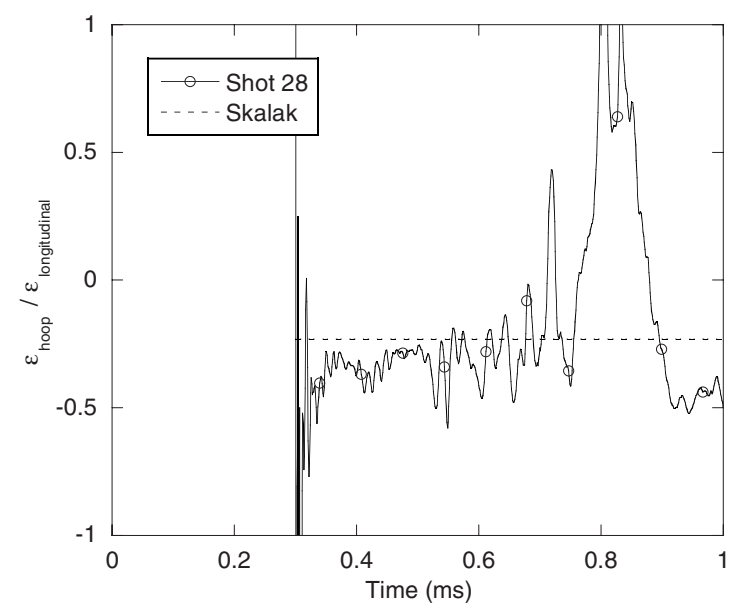

Fig. 7 Ratio between hoop and longitudinal strains of Fig. 6

[6] models of the buffer-water dynamics predicts an exponential decay of pressure behind the initial peak. Differences in acoustic impedance between the impactor, buffer, and fluid as well as the deformation of the thin-walled tubes result in a less ideal situation for the present experiments than considered by the previous authors; as a consequence, prediction of the pressure pulse is less straightforward.

Figure 7 shows the time history of the ratio of the longitudinal strain to hoop strain from Fig. 6. Skalak's theoretical prediction is that this ratio is independent of the peak pressure. For the thinwalled tubes, the ratio is predicted to be $\varepsilon_{\text {longitudinal }} / \varepsilon_{\text {hoop }}=$ -0.230 , which is also plotted in the figure. The ratio in the experiment strongly fluctuates but is comparable to the prediction except right at the wave front. The oscillations in the pressure signals can arise from a variety of effects, such as pressure waves generated during the impact process and radial oscillations of the tube wall and fluid.

3.2 Plastic Waves in Thin Tubes. As the buffer speed is increased, the peak amplitude of the initial strain wave is observed to exceed the nominal elastic-plastic proportional limit of $2 m$ strain. As an example of this, the hoop strain and pressure histories in shot 29, and the longitudinal strains are shown in Fig. 8 . The precursor and primary wave velocities are $5342 \mathrm{~m} / \mathrm{s}$ and $1237 \mathrm{~m} / \mathrm{s}$, respectively. Although Skalak's phase velocities are obtained by assuming elastic behavior, the experiments are in reasonable agreement with Skalak's model: $c_{2}=5260 \mathrm{~m} / \mathrm{s}$ and $c_{1}$ $=1199 \mathrm{~m} / \mathrm{s}$.

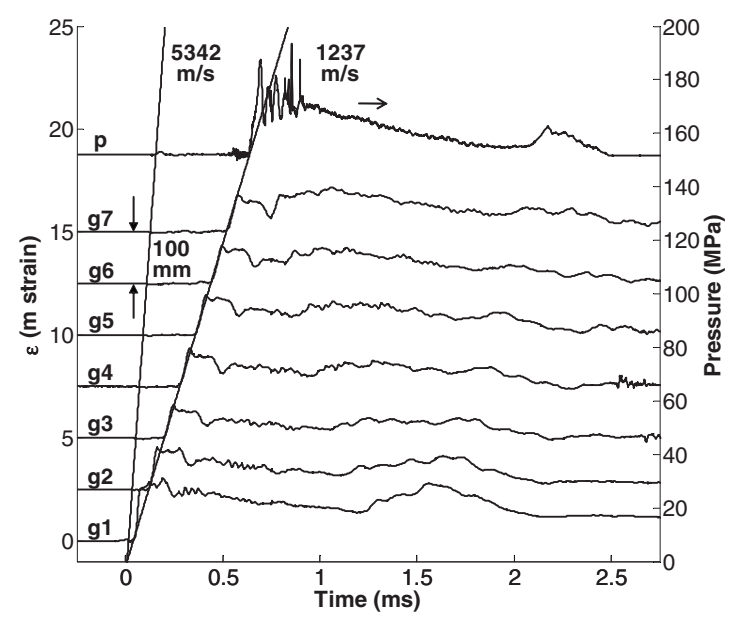

Fig. 8 Hoop strain and end pressure histories-plastic waves in shot $29, V_{B}=16.6 \mathrm{~m} / \mathrm{s}\left(V_{P}=19.3 \mathrm{~m} / \mathrm{s}, P_{D}=0.65 \mathrm{MPa}\right)$ 


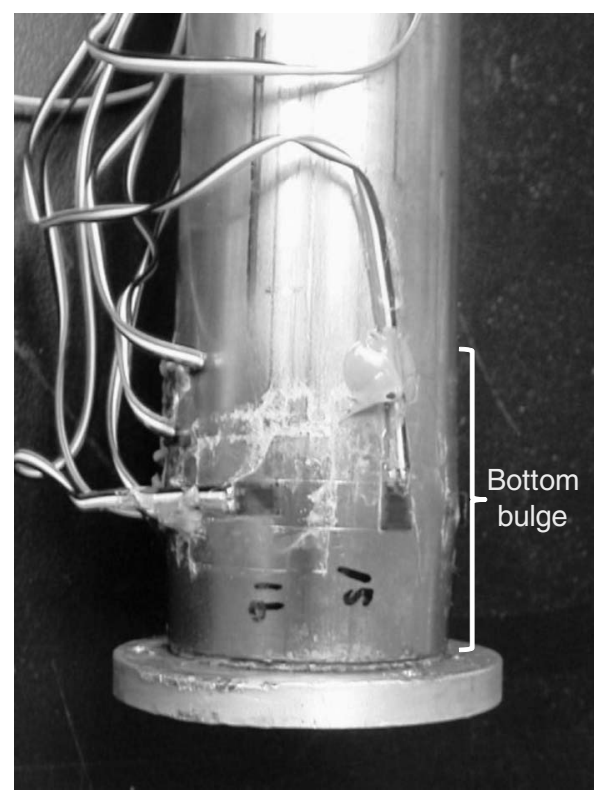

Fig. 9 Bulge near the closed-end bottom created by the reflection of the stress wave

The strain history at g1 (the nearest location to the surface of the buffer) indicates that the peak amplitude of the hoop strain is larger than $3.0 \mathrm{~m}$ strain and gradually reduces until at g7, the value is less than $2.0 \mathrm{~m}$ strain. Since the loading is dynamic and the yield strength of mild-steel is a strong function of strain rate, the onset of yielding is expected to occur at strains higher than $2.0 \mathrm{~m}$ strain. A visible bulge at the bottom of the specimen tube confirmed that plastic deformation definitely occurred in experiments with higher buffer speeds, as shown in Fig. 9. These bulges are associated with high pressure created by the reflection of the stress waves at the tube bottom. A visible bulge is also observed near the top of the specimen tube, at the location of the buffer bottom surface. Examination of the strain traces and measurement of the strain following the test also provides evidence of plastic deformation (Fig. 10). The residual hoop strains are $0.16 \mathrm{~m}$ strain and $1.2 \mathrm{~m}$ strain at g5 and $\mathrm{g} 1$, respectively.

A closeup of hoop and longitudinal strain histories in shot \#29

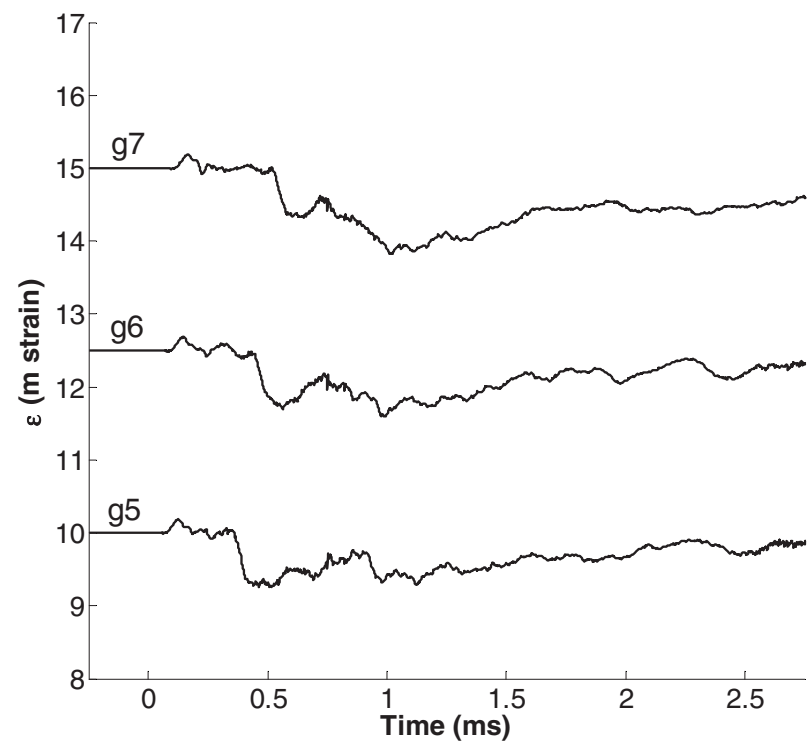

Fig. 10 Longitudinal strain histories-shot 29

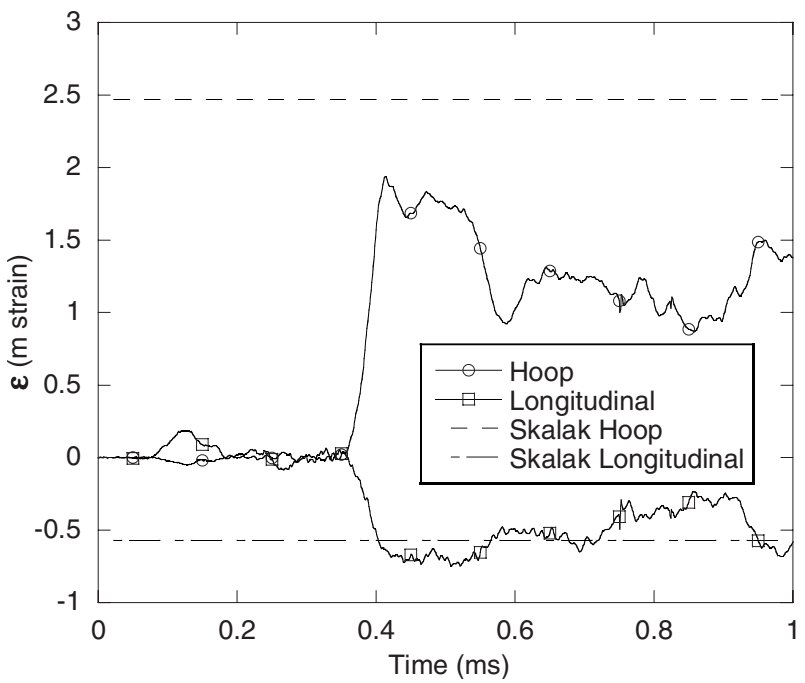

Fig. 11 Hoop and longitudinal strain histories-plastic waves in shot 29, gauge location $5, V_{B}=16.6 \mathrm{~m} / \mathrm{s}\left(V_{P}=19.3 \mathrm{~m} / \mathrm{s}, P_{D}\right.$ $=0.65 \mathrm{MPa}$ )

at g5 is shown in Fig. 11. Skalak's predictions for hoop and longitudinal strains are $2.47 \mathrm{~m}$ strain and $-0.569 \mathrm{~m}$ strain with the buffer speed $V_{B}$. Since averaged hoop and longitudinal strains measured at 7 gauges are $2.02 \mathrm{~m}$ strain and $-0.744 \mathrm{~m}$ strain (see Table 4), the predicted hoop strain is larger than the experiment while the longitudinal strain is smaller.

In the plastic case, the magnitude of hoop and longitudinal strains increases and the ratio fluctuates less than in the elastic case (see Fig. 12). The peak hoop strain is larger than the predicted value at $\mathrm{g} 1$, and then becomes much smaller at $\mathrm{g} 5$ while the peak longitudinal strain is still close to the predicted value. The dissipation of energy due to plastic deformation results in the decay of the peak amplitude between g1 and g5. By comparison, in the purely elastic cases, the wave amplitude remains relatively constant in propagation from $\mathrm{g} 1$ and $\mathrm{g} 7$.

3.3 Comparison Between Theory and Experiments. Although some plastic deformation is observed for higher buffer speeds, the maximum residual strain is still smaller than $2.02 \mathrm{~m}$ strain except near the bottom end of the specimen. For this reason, we believe that our experimental results can be compared with the classical elastic theory although some deviation should

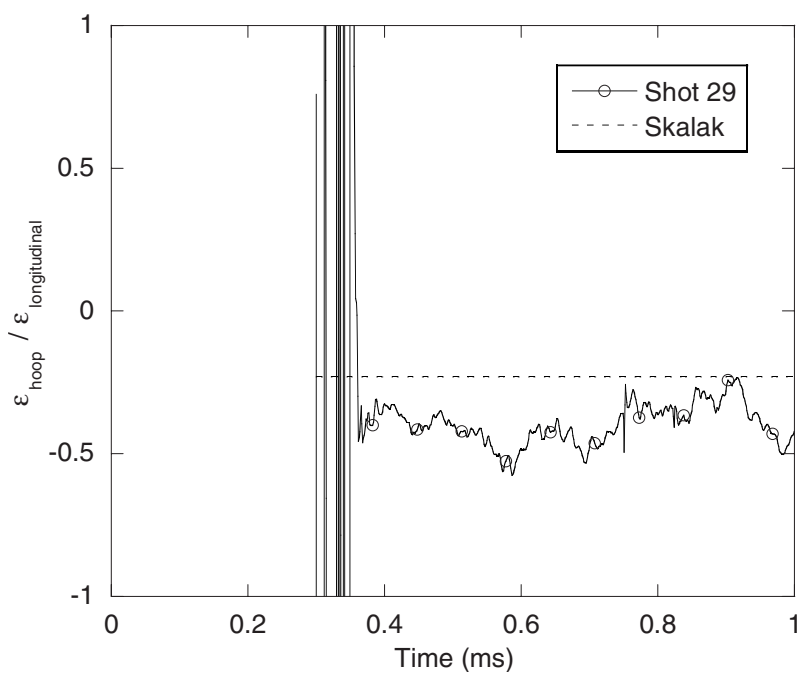

Fig. 12 Ratio between hoop and longitudinal strains of Fig. 11 


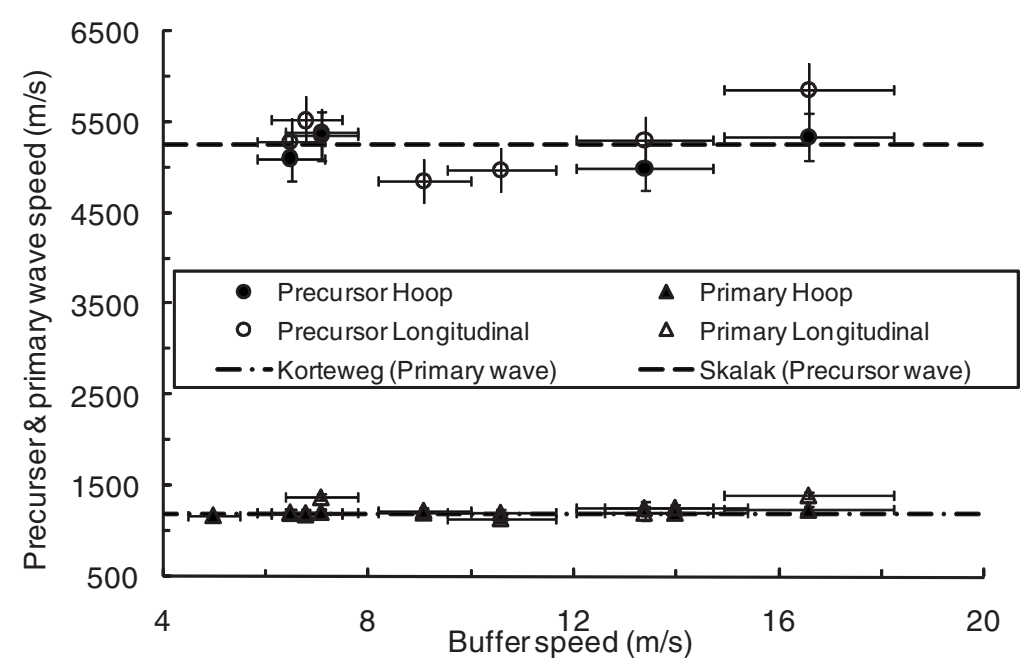

Fig. 13 Precursor and primary wave speeds versu buffer speeds for specimen tubes $1-3$

be expected at the higher projectile speeds. Precursor and primary wave speeds in thin-walled tubes (tubes 1-3) are plotted versus the maximum buffer speeds in Fig. 13. The predicted phase velocities show good agreement with the present experimental results: $c_{1}=1199 \mathrm{~m} / \mathrm{s}, c_{2}=5260 \mathrm{~m} / \mathrm{s}$, independent of the buffer speed.

Averaged peak hoop and longitudinal strains are plotted in Fig. 14. The experimental results show reasonable agreement with theoretical predictions with the uncertainty range of most points overlapping the predictions. As discussed Sec. 3.2, the primary wave front strains are oscillatory and this leads to a large uncertainty in the observed peak strain.

3.4 Thick-Walled Tube Results. Watters [22] concluded that the use of the thick-wall equations is important when the ratio of the inner diameter to the wall-thickness $(2 a / h)$ is less than 40 . The ratio $2 a / h$ of the thin-walled specimen tubes discussed in Secs. 3.1-3.3 is over 52. In this section, we present the results of tests carried out with $h=6.4$ and $12.7 \mathrm{~mm}$ thick-walled specimens. Since the $h=6.4 \mathrm{~mm}$ tube gave the same qualitative results as the $h=12.7 \mathrm{~mm}$ tube, we only discuss the latter case. Figure 15 shows the hoop-strain histories in shot 62 at $V_{B}=15.2 \mathrm{~m} / \mathrm{s}$. We previously observed plastic waves propagating through the thinwalled tube at a similar buffer speed of $16.6 \mathrm{~m} / \mathrm{s}$. Since the interaction between the tube and water is quite weak in this case, the amplitudes of the elastic waves are a factor of 10 smaller than for

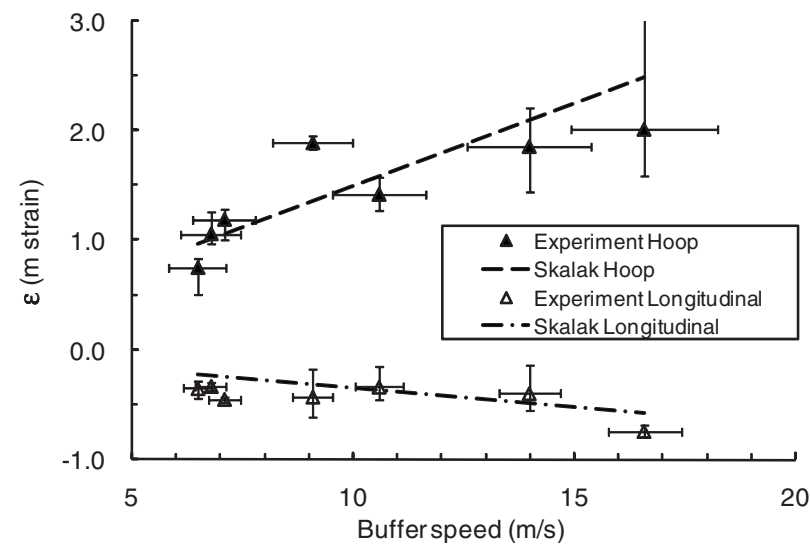

Fig. 14 Averaged peak hoop and longitudinal strains vs buffer speeds for specimen tubes $1-3$ the thin-walled tubes and the primary wave propagates at 1486 $\mathrm{m} / \mathrm{s}$, which is very close to the water sound speed. Figure 16 shows the longitudinal strain histories in the same format as Fig. 15. As the wall-thickness increases, the longitudinal wave behaves more dispersively and has less correlation to the hoop wave.

Pressure transducers were mounted in the wall of tube 5 at locations g1, g4, and g6. The pressure signals (Fig. 17) show the initial wave generated by the impact of the projectile, propagation through water, and reflection from the bottom boundary. After the arrival of the reflected wave at the buffer, the buffer moves upward, which produces a tension wave. This is observed at g1 as a period of nearly constant, negative pressure after $1.5 \mathrm{~ms}$. This tension wave propagates and appears subsequently at g4 and g6. A reflected wave can be observed propagating back through the tension region at $2 \mathrm{~ms}$ on $\mathrm{g} 4$ but by the time it reaches $\mathrm{g} 1$ at $2.3 \mathrm{~ms}$, it has been significantly attenuated. The appearance of negative pressure regions and the association with cavitation is well established by previous studies $[4,15]$.

We compare measured and predicted primary wave speeds as a function of the wall-thickness in Fig. 18. As anticipated from Eq. (1), wave speeds increase as the wall-thickness increases. The experimental values show reasonable agreement with both

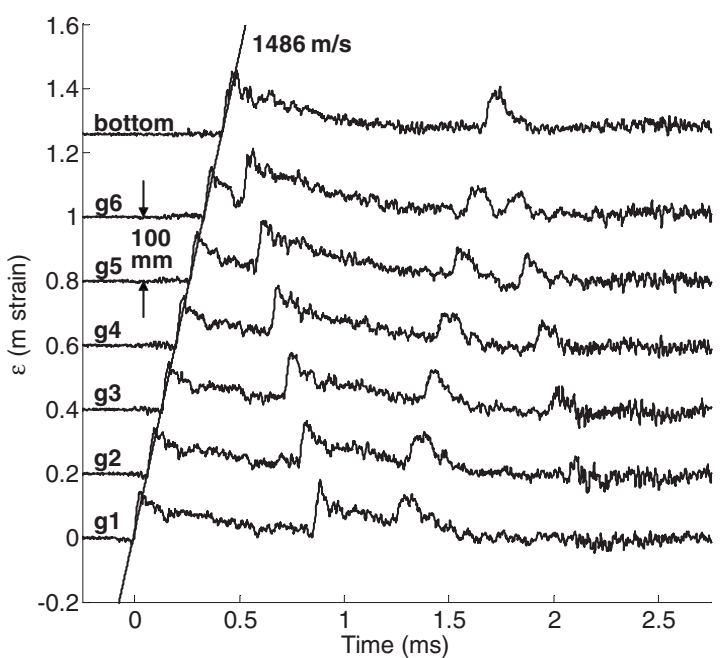

Fig. 15 Hoop-strain histories in shot 62 with specimen tube 5 $\left(12.7 \mathrm{~mm}\right.$ thick wall), $V_{B}=15.2 \mathrm{~m} / \mathrm{s} \quad\left(V_{P}=18.5 \mathrm{~m} / \mathrm{s}, P_{D}\right.$ $=0.65 \mathrm{MPa})$ 


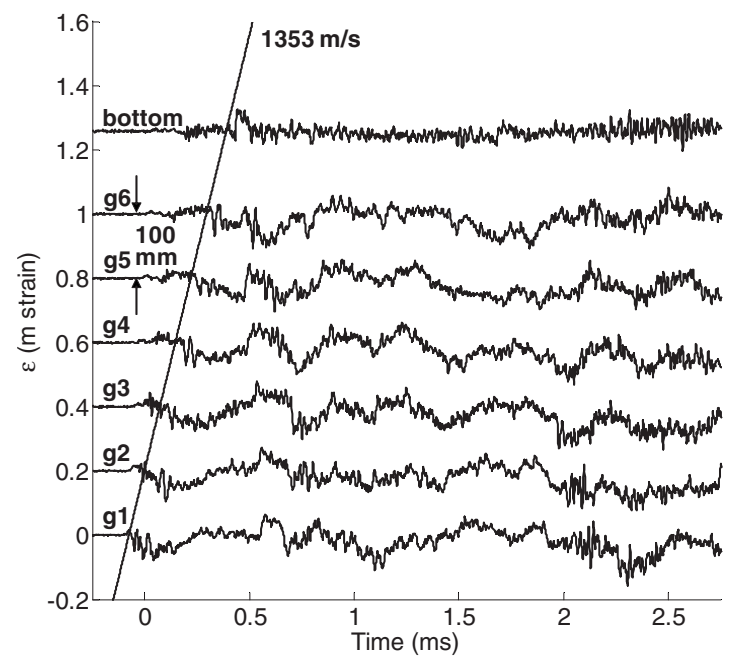

Fig. 16 Longitudinal strain histories in shot 62 with specimen tube 5 (12.7 mm thick wall), $V_{B}=15.2 \mathrm{~m} / \mathrm{s}\left(V_{P}=18.5 \mathrm{~m} / \mathrm{s}, P_{D}\right.$ $=0.65 \mathrm{MPa}$ )

Korteweg's approximate thin-wall theory and Tijsseling's thickwall [23] theory.

We can experimentally examine the relationship between peak pressure and peak hoop strain by using the measured values of



Fig. 17 Side wall pressure histories at locations 1, 4, and 6 in shot 62 with specimen tube $5\left(12.7 \mathrm{~mm}\right.$ thick wall), $V_{B}$ $=15.2 \mathrm{~m} / \mathrm{s}\left(V_{P}=18.5 \mathrm{~m} / \mathrm{s}, P_{D}=0.65 \mathrm{MPa}\right)$

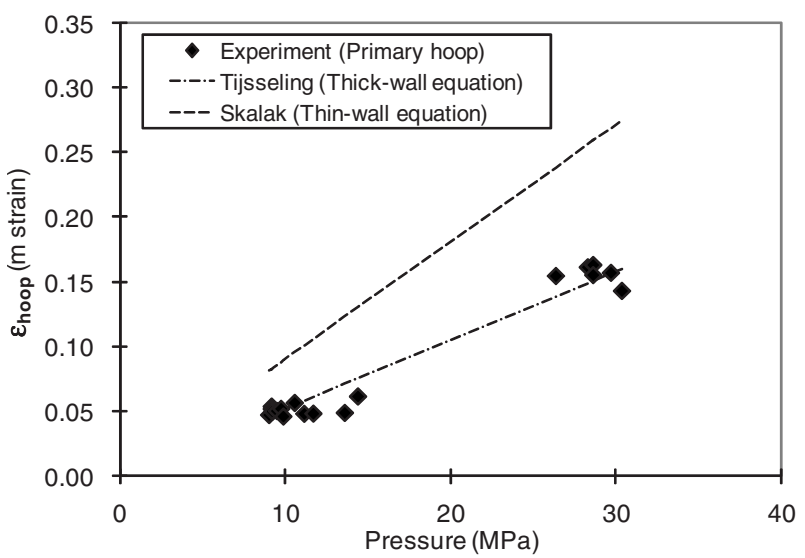

Fig. 19 Primary hoop strain versus side-wall pressure for 12.7 $\mathrm{mm}$ thick-walled tube

both parameters. Substituting $a=25.4 \mathrm{~mm}$ and $h=12.7 \mathrm{~mm}$ into Skalak's theoretical expressions [14], we obtain the relationship for hoop strain in terms of the parameter $p_{0}$ :

$$
\varepsilon_{\text {hoop }}=0.962 \frac{p_{0} a}{E h}
$$

Here $a$ is the average of the inner and outer radii of tube 5. The pressure associated with the primary wave is $p=0.979 p_{0}$ according to Skalak's theory. Tijsseling [23] developed a correction for the thick-walled tubes by assuming a quasistatic stress distribution across the thickness of the pipe wall. He obtained the hoop strain at the external surface tube to be

$$
\varepsilon_{\text {hoop,Tijsseling }}=\frac{1}{E}\left(\frac{a}{h} \frac{1}{1+\frac{1}{2} \frac{h}{a}}\left(p-p_{\text {out }}\right)-(1-\nu) p_{\text {out }}\right)
$$

where $p$ and $p_{\text {out }}$ are the internal and external (ambient) pressures. We compare the experimental and theoretical results in Fig. 19. The experimental results agree well with the thick-wall approximation of Tijsseling and clearly disagree with Skalak's thin-wall model in this case. The correction for wall-thickness is clearly more important for predicting the strain-pressure relationship than the wave speed.

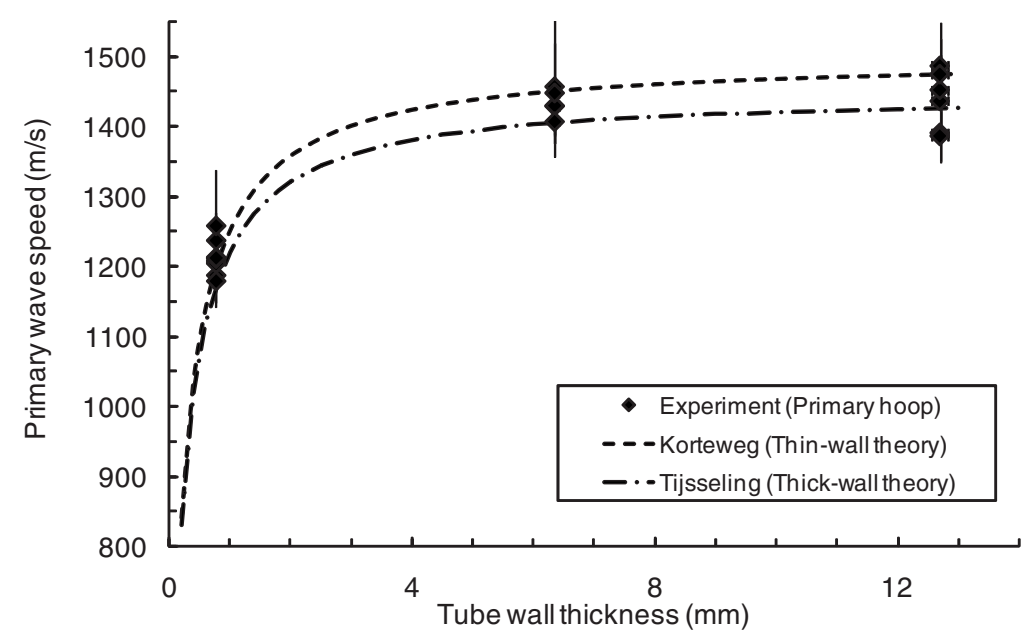

Fig. 18 Primary hoop stress wave speeds vesus wall-thickness of specimen tubes 


\section{Conclusion}

We have used projectile impact and steel tubes filled with water to study the propagation of coupled structural and pressure waves. We are able to use much smaller test rigs than typically employed in water hammer studies through the use of high-speed instrumentation and projectile impact.

The predictions of the classical theory of Skalak agree reasonably well with our observations for the case of the thin-walled tubes and elastic motions. We find qualitative agreement with the predicted splitting of the wave into precursor and primary branches with two distinct wave speeds. We obtain quantitative agreement at the level of $10 \%$ maximum discrepancies between theory and experiment for both peak amplitude and wave speeds.

The range of wave amplitudes that can be examined is limited on the upper end by plastic deformation and on the lower end by the sensitivity of the instrumentation. We have varied the amplitude of the pressure and over a range of about 3 and strain over a range of 20 in the present study. A significant amount of damping is observed following the onset of plastic deformation and the amplitude of the peak strain is observed to decay substantially as the wave propagates along the tube.

The variation in wave speed and amplitude with wall-thickness is in reasonable agreement with the theoretical predictions. For thick-walled tubes, the peak strain is substantially reduced due to through-wall stresses, in agreement with the approximate thickwall model of Tijsseling [23].

\section{Acknowledgment}

This research was sponsored by the Office of Naval Research, DOD MURI on Mechanics and Mechanisms of Impulse Loading, Damage and Failure of Marine Structures and Materials (ONR Grant No. N00014-06-1-0730), program manager Dr. Y.D.S. Rajapakse. We thank Chris Krok for his work on the first generation of experiments and Tim Curran for his work on data processing and image analysis.

\section{Nomenclature}

$$
\begin{aligned}
a & =\text { radius of pipe } \\
c & =\text { velocity of sound in water } \\
c_{0} & =\text { velocity of sound in tube wall } \\
c_{1}, c_{2} & =\text { Skalak's phase velocities } \\
c_{K} & =\text { Korteweg's phase velocity } \\
E & =\text { Young's modulus of tube material } \\
h & =\text { thickness of tube wall } \\
K & =\text { bulk modulus of fluid } \\
m & =\text { mass of tube per unit surface area } \\
P_{D} & =\text { driver (reservoir) pressure }
\end{aligned}
$$

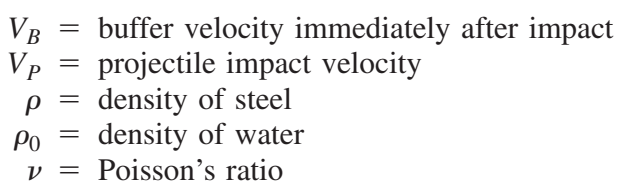

\section{References}

[1] Cole, R., 1965, Underwater Explosions, Dover, New York.

[2] UER, 1950, "Underwater Explosion Research: A Compendium of the British and American Reports, Vol. I-The Shock Wave, Vol. II-The Gas Globe, Vol. III-The Damage Process," Office of Naval Research Technical Report.

[3] Kedrinsky, V., 2005, Hydrodynamics of Explosion, Springer, New York.

[4] Trevena, D., 1987, Cavitation and Tension in Liquids, Hilger, London.

[5] Skews, B., Kosing, E., and Hattingh, R., 2004, "Use of a Liquid Shock Tube as a Device for the Study of Material Deformation Under Impulsive Loading Conditions," Proc. Inst. Mech. Eng., Part C: J. Mech. Eng. Sci., 218, pp. $39-51$.

[6] Deshpande, V. S., Heaver, A., and Fleck, N. A., 2006, "An Underwater Shock Simulator,” Proc. R. Soc. London, Ser. A, 462, pp. 1021-1041.

[7] Espinosa, H., Lee, S., and Moldovan, N., 2006, "A Novel Fluid Structure Interaction Experiment to Investigate Deformation of Structural Elements Subjected to Impulsive Loading," Exp. Mech., 46, pp. 805-824.

[8] Korteweg, D., 1878, "Über die fortphlanzungsgeschwindigkeit des schalles in elastisches rohren," Ann. Phys. Chem., 241, pp. 525-542.

[9] Joukowsky, N., 1900, "Über den hydraulischen stoss in wasserleitungsrohren," St. Petersburg: Memoires de l'Aacademie Imperiale des Sciences, 8th Series, Vol. IX, No. 5.

[10] Wiggert, D. C., and Tijsseling, A. S., 2001, "Fluid Transients and FluidStructure Interaction in Flexible Liquid-Filled Piping," Appl. Mech. Rev., 54(5), pp. 455-481.

[11] Fuller, C. R., and Fahy, F. J., 1982, "Characteristics of Wave Propagation and Energy Distributions in Cylindrical Elastic Shells Filled With Fluid," J. Sound Vib., 81, pp. 501-518

[12] Sinha, B. K., Plona, T. J., Kostek, S., and Chang, S.-K., 1992, “Axisymmetric Wave Propagation in Fluid-loaded Cylindrical Shells," J. Acoust. Soc. Am., 92, pp. 1132-1143.

[13] Pinnington, R. J., 1997, "The Axisymmetric Wave Transmission Properties of Pressurized Flexible Tubes," J. Sound Vib., 204, pp. 271-289.

[14] Skalak, R., 1956, "An Extension to the Theory of Water Hammer," Trans. ASME, 78, pp. 105-116.

[15] Wylie, E. B., and Streeter, V. L., 1993, Fluid Transients in Systems, PrenticeHall, Inc., Englewood Cliffs, NJ.

[16] Tijsseling, A. S., 1996, "Fluid-Structure Interaction in Liquid-Filled Pipe Systems: A Review," J. Fluids Struct., 10, pp. 109-146.

[17] Tijsseling, A. S., 2003, "Exact Solution of Linear Hyperbolic Four-Equation System in Axial Liquid-Pipe Vibration," J. Fluids Struct., 18, pp. 179-196.

[18] Li, Q. S., Yang, K., and Zhang, L., 2003, "Analytical Solution for FluidStructure Interaction in Liquid-Filled Pipes Subjected to Impact-Induced Water Hammer," J. Eng. Mech., 129(12), pp. 1408-1417.

[19] Tijsseling, A. S., Lambert, M. F., Simpson, A. R., Stephens, M. L., Vítkwský, J. P., and Bergant, A., 2008, "Skalak's Extended Theory of Water Hammer," J. Sound Vib., 310, pp. 718-728.

[20] Meyers, M. A., 1994, Dynamic Behavior of Materials, Wiley, New York

[21] Fowles, G. R., 1960, "Attenuation of a Shock Wave Produced in a Solid by a Flying Plate," J. Appl. Phys., 31, pp. 655-661.

[22] Watters, G. Z., 1984, Analysis and Control of Unsteady Flow in Pipelines, Butterworth, Washington, DC.

[23] Tijsseling, A. S., 2007, "Water Hammer With Fluid-Structure Interaction in Thick-Walled Pipes," Comput. Struct., 85, pp. 844-851. 